\title{
WIRELESS WEATHER STATION USING ARDUINO MEGA AND ARDUINO NANO
}

\author{
A.C. GHEORGHE ${ }^{1}$, C.I. STOICA ${ }^{2}$ \\ Valahia University of Targoviste, Faculty of Electrical Engineering, Electronics and Information Technology \\ E-mail: gheorghe_andrei89@yahoo.com
}

\begin{abstract}
The study aims for the development of a wireless weather station composed of two modules, the outdoor module that takes the temperature and humidity from the environment through the DHT22 sensor and transmits the information through the n24RFL01+ communication module to the indoor module. The indoor module takes the temperature and humidity from the environment and displays it on a 3.5" TFT display along with the information received from the outdoor module, also the date and time are displayed. The development boards used for the weather station are Arduino Mega 2560 for the indoor module and Arduino Nano for the outdoor module. The n24RFL01+ wireless communication module, depending on the model, can transmit data at a distance of $800+m$ and the DHT22 sensor is very accurate. The programming code used for the development of the weather station is made in Arduino IDE. Arduino IDE is an open-source software that is used to write and upload code to the Arduino developing boards.
\end{abstract}

Keywords: Arduino, Weather station, Wireless

\section{INTRODUCTION}

The weather station is a device that can measure the parameters of the weather based on atmospheric conditions both on land and sea for an intended location using devices that are specific for forecasting the weather condition and studying different climatic properties. The concerns related with the prediction of the weather officially started in the nineteenth century, after that the basis of recording and measuring the weather conditions in certain places changed. The data that is collected allows for the decision and for the confirmation of the selected location. The weather is largely made from two components, humidity and temperature. These components can vary especially in places that are afflicted by the temperature of solar radiation and the locations based on the tropical line [1]. The weather station proposed for this paper is composed of two modules, the indoor module that measures the indoor temperature and humidity through the DHT22 sensor and the outdoor module that measures the outdoor temperature and humidity through the DHT22 sensor. The information measured by both modules is displayed on the built-in 3.5" TFT display of the indoor module. The built-in display also offers the user information about the current date and time. Communication between the two Arduino development boards (indoor module - Arduino Mega, outdoor module Arduino Nano) is achieved through the wireless module $\mathrm{n} 24 \mathrm{RFL} 01+$ running at an ISM frequency (industrial, scientific and medical) between $2.4 \mathrm{GHz}$ and $2.5 \mathrm{GHz}$. The
DHT22 sensor is very accurate and can measure the temperature between $-40{ }^{\circ} \mathrm{C}$ and $80^{\circ} \mathrm{C}$ with an error of \pm 0.5 ${ }^{\circ} \mathrm{C}$ and the relative humidity between $0 \%$ and $100 \%$ with an error of $2 \%-5 \%$.

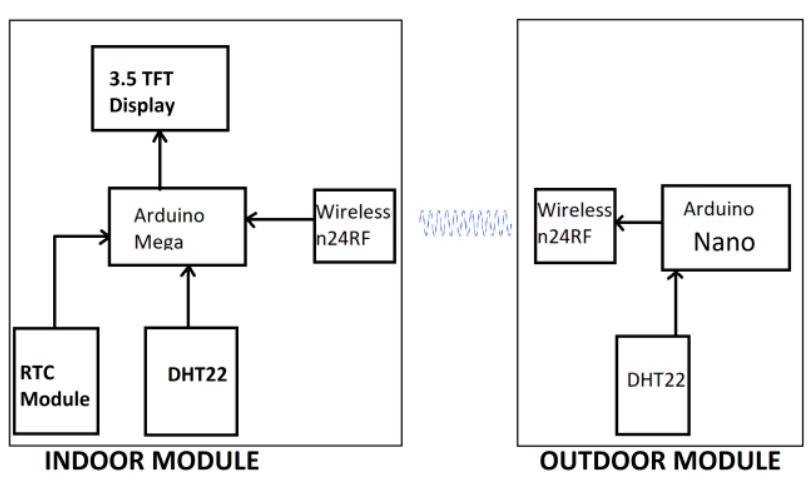

Figure 1. System block diagram.

In Figure 1 we can see the block diagram of the weather station. The indoor module is composed from the Arduino Mega developing board, wireless n24RF module, 3.5" TFT display, RTC module and the DHT22 sensor. The outdoor module is composed from the Arduino Nano developing board, wireless n24RF module and the DHT22 sensor. Each module is powered by a $9 \mathrm{~V}$ battery.

\subsection{Arduino Nano}

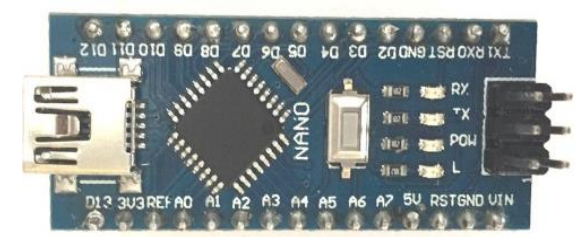

Figure 2. Arduino Nano developing board.

The Arduino Nano development board (e.g., Figure 2) is one of the smallest development boards in the Arduino family when it comes to size. The small size of this development board ensures the development of applications that are required to have a small displacement. As technical specifications, the Arduino nano development board is based on the Atmega328P microcontroller, which is developed on the Atmel AVR architecture. Arduino Nano has 12 digital input / output ports they find D2 - D13, 8 analog input ports they find A0 - A7, 1 pair of serial transceiver ports at TTL RX / TX level, 6 PWM ports, D3, D5, D6, D9, D10, D11. The development board supports power using the USB port, external power supply of $5 \mathrm{~V}-12 \mathrm{~V} \mathrm{DC}$ or $9 \mathrm{~V}$ power battery. The flash memory of the development board is 32 
$\mathrm{KB}$, the RAM memory of the SRAM type is $2 \mathrm{~KB}$ EEPROM ROM is $1 \mathrm{~KB}$.

\subsection{Arduino Mega 2560}

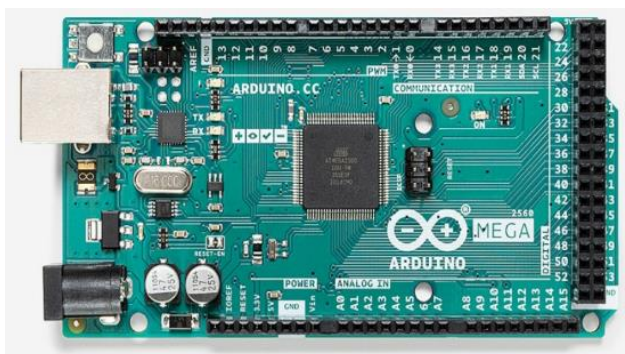

Figure 3. Arduino Mega developing board.

The Arduino Mega 2560 (e.g., Figure 3) is a development board that has the ATMega2560 microcontroller. The development board comes with greater space for memory and more I/O pins than other developing boards found on the market. The digital I/O pins are 54 in total and the developing board comes with 16 analog pins which makes this board more attractive for different complex applications. From the 54 digital I/O pins, 15 are used for PWM (Pulse Width Modulation) [3]. The development board has a crystal oscillator that has a frequency of $16 \mathrm{MHz}$ and comes equipped with a USB port that is used to transfer the source code created in the IDE program. The D.C power port is used to power the development board, some development boards such as the Arduino Pro mini do not come with a power port. The additional ICSP port present on the development board can be used to program the board [4]. The development board comes with 2 voltage stabilizers ( $5 \mathrm{~V}$ and $3.3 \mathrm{~V}$ ) which offers the possibility to supply various components, supports a power supply between $6 \mathrm{~V}$ and $20 \mathrm{~V}$, flash memory of 256 $\mathrm{KB}$, SRAM type RAM of $8 \mathrm{~KB}$, ROM memory type EEPROM of $4 \mathrm{~KB}$ and has a size of $101 \times 53 \mathrm{~mm}$.

\section{3 n24RFL01+ wireless module}

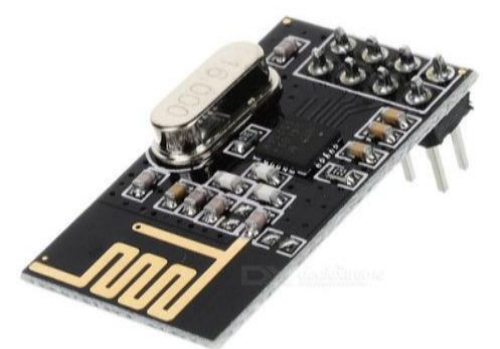

Figure 4. n24RFL01+.

The nRF24L01+ wireless module (e.g., Figure 4) is a radio transceiver that has a single chip that uses the ISM (industrial, scientific and medical) band at a frequency of $2.4 \mathrm{GHz}-2.5 \mathrm{GHz}$. The module is made from a built-in frequency synthesizer, power amplifier, crystal oscillator, modulator, demodulator and has the Enhanced ShockBurst protocol [2]. The output power, communication channel and the protocol configuration are programmed from the SPI (Serial Peripheral
Interface). The power consumption is $9.0 \mathrm{~mA}$ at an output power of $-6 \mathrm{dBm}$ and $12,3 \mathrm{~mA}$ in RX mode. The power down mode and the sleep mode makes the module very efficient. The maximum data rate is $2 \mathrm{Mb} / \mathrm{s}$ and the output power is $0 \mathrm{dBm}$. The module operates within a voltage range of $1.9 \mathrm{~V}-3.6 \mathrm{~V}$ and the range of communication depending on the model is $800+\mathrm{m}$ in line of sight.

\subsection{RTC module}

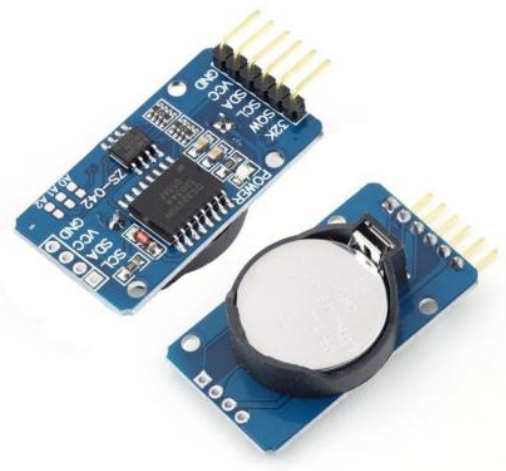

Figure 5 RTC module.

The RTC module (e.g. Figure 5) is basically a system that stores the date and the time, this module has a battery that keeps the module turned on when is no external power thus keeping the date and time up to date. The RTC module has a DS3231 RTC chip, this chip manages all the date/time functions and has a i2c interface which can be connected with any microcontroller. The chip stores information about the seconds, minutes, hours, days, months and years including the date correction for leap years until 2100. The time is stored in 24- or 12-hour format with a AM/PM indication and has two alarms that can be programmed during the day. The RTC module also has a SQW pin that provides a square wave through programming with a value of $1 \mathrm{kHz}, 4 \mathrm{kHz}, 8 \mathrm{kHz}$ or 32 $\mathrm{kHz}$, this function can be used as an interrupt function in many applications based on time. The DS3231 is powered by a $32 \mathrm{kHz}$ crystal oscillator, the crystal oscillator is immune to external temperature changes.

\subsection{DHT22 sensor}

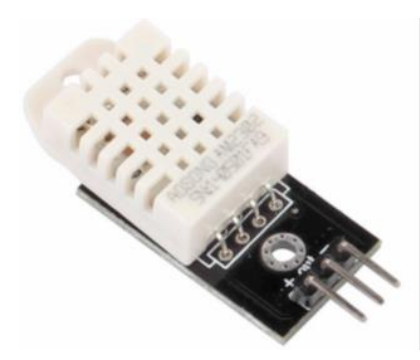

Figure 6. DHT22 sensor.

The DHT22 sensor is an economical digital sensor that measures the temperature and the humidity. The sensor uses a capacitive humidity sensor and a thermistor that measure the ambient air. The sensor provides a digital 
signal thus no analog input is required. The voltage required for operating the sensor is between $3 \mathrm{~V}$ and $5 \mathrm{~V}$ with a power consumption of $2.5 \mathrm{~mA}$. The sensor can measure the temperature between $-40^{\circ} \mathrm{C}$ and $80^{\circ} \mathrm{C}$ with an error of $\pm 0,5^{\circ} \mathrm{C}$ and the humidity between $0 \%$ and $100 \%$ with an error of $2-5 \%$.

\section{THE ELECTRONIC BOARD}

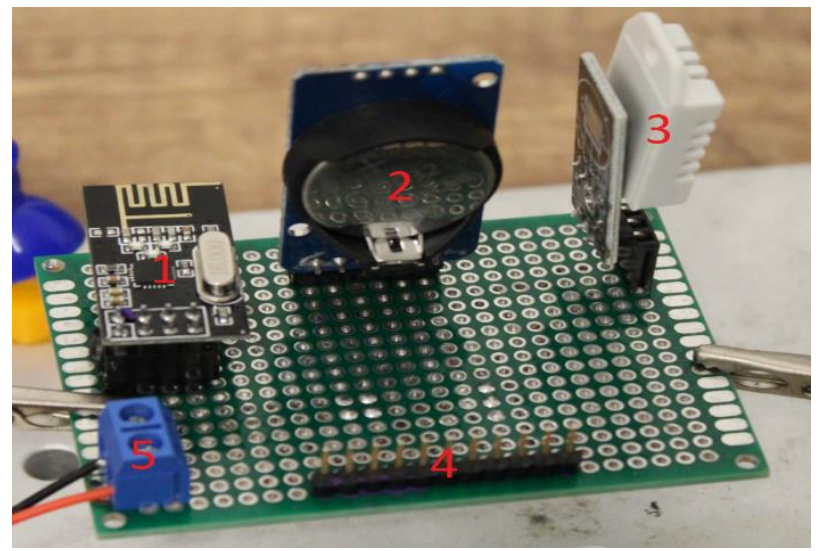

Figure 7. Indoor module electronic board.

The indoor module electronic board (e.g., Figure 7) is made from the n24RFL01+ module (1), RTC module (2), DHT22 sensor (3), power connector (5) and Arduino Mega pin connection header. The pin connection header is used to connect the electronic board to the Arduino Mega developing board for data transfer and power supply. The electronic board with the Arduino Mega and the 3.5 " TFT display are power by a $9 \mathrm{~V}$ battery.

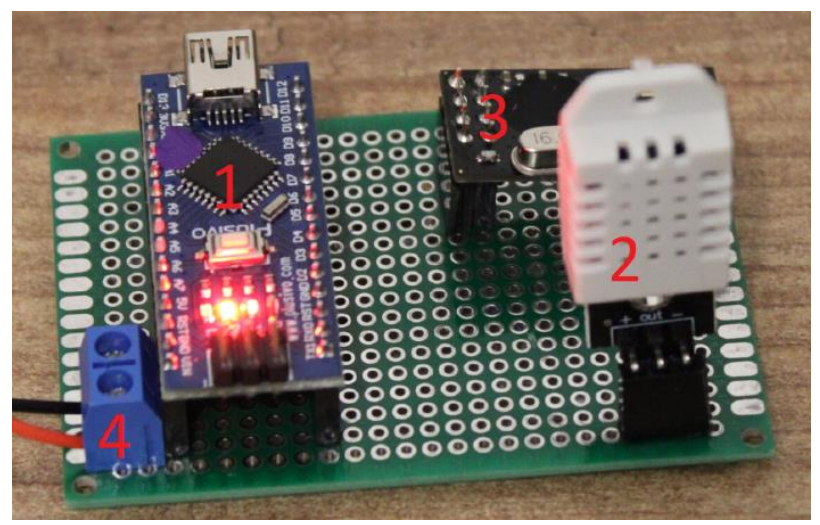

Figure 8. Outdoor module electronic board.

The outdoor module electronic board (e.g., Figure 8) is made from the Arduino Nano developing board (1), DHT22 sensor (2), n24RFL01+ module (3) and the power connector (4). The electronic board is powered by a $9 \mathrm{~V}$ battery.

\section{THE FUNCTIONING PRINCIPLE}

The outdoor module measures the temperature and the humidity of the outdoor ambient air with the help of the DHT22 sensor then the measured data is processed by the Arduino Nano developing board that sends it trough the $\mathrm{n} 24 \mathrm{RFL} 01+$ to the indoor module. The indoor module measures the temperature and the humidity of the indoor ambient air with the help of the DHT22 sensor then the measured data is processed by the Arduino Mega developing board along with the data received from the n24RFL01+ module and displays it on the 3.5" TFT display with the current date and time.

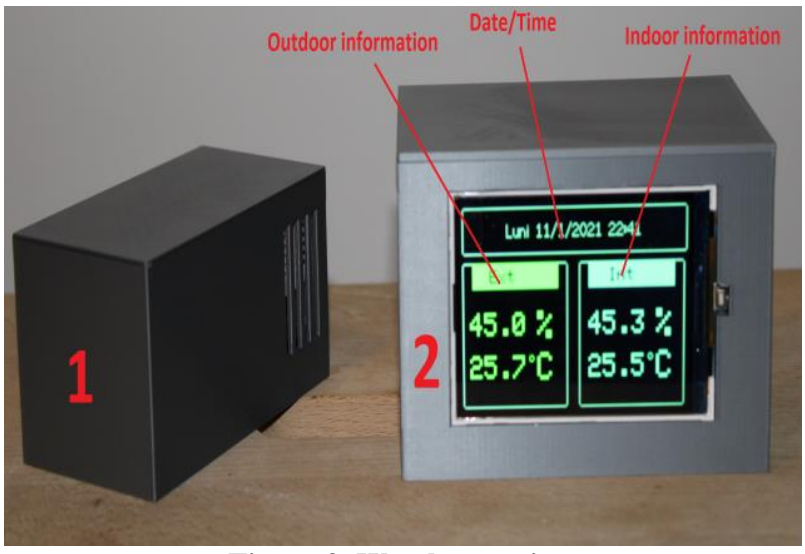

Figure 9. Weather station.

In Figure 9 we have the weather station fully assembled. The outdoor module (1) is connected to the indoor module (2) thus displaying similar information about the measured temperature/humidity and the date/time on the 3.5 " TFT display [5].

\section{CONCLUSIONS}

The paper demonstrates the design and implementation of a wireless weather station for monitoring environmental parameters. The weather station consists of two modules that are used to monitor indoor and outdoor temperature and humidity. The indoor module displays the temperature / humidity recorded by both modules and the date / time on the 3.5 " TFT display. The outdoor module communicates with the inner module via the n24RFL01+ wireless transmitter. The output power, frequency channels and protocol configuration of the wireless transmitter are easily programmable via an SPI interface and allows depending on the n24RFL01+ transmitter used to communicate over a distance of up to $800+\mathrm{m}$ in the line of sight. The DHT22 temperature and humidity sensor used can measure the temperature between $-40^{\circ} \mathrm{C}$ and $80^{\circ} \mathrm{C}$ with an error of $\pm 0,5^{\circ} \mathrm{C}$ and the humidity between $0 \%$ and $100 \%$ with an error of $2-5 \%$.

\section{REFERENCES}

[1] Stephen Burt, The Weather Observer's Handbook, Cambridge University Press, 2012.

[2] Robert Sobot, Wireless Communication Electronics: Introduction to RF Circuits and Design Techniques, Springer Nature, 2020.

[3] John Boxall, Arduino Workshop, No Starch press, 2013. 
[4] Jeremy Blum, Exploring Arduino: Tools and Techniques for Engineering Wizardry, John Wiley \& Sons, 2013.

[5] Simon Monk, Programming Arduino: Getting Started with Sketches, McGraw Hill Professional, 2016. 